\title{
Lie Group Analysis on Brownian Motion and Thermophoresis Effect on Free Convective Boundary-Layer Flow on a Vertical Cylinder Embedded in a Nanofluid-Saturated Porous Medium
}

\author{
Mohammad Ferdows, ${ }^{1}$ Mohammed Abdul Ali Hamad, ${ }^{2}$ and Mohamed Ali ${ }^{3}$ \\ ${ }^{1}$ Department of Mathematics, University of Dhaka, Dhaka 1000, Bangladesh \\ ${ }^{2}$ Mathematics Department, Faculty of Science, Assiut University, Assiut 71516, Egypt \\ ${ }^{3}$ Mechanical Engineering Department, King Saud University, P.O. Box 800, Riyadh 11421, Saudi Arabia
}

Correspondence should be addressed to Mohamed Ali; mali@ksu.edu.sa

Received 21 January 2014; Revised 23 June 2014; Accepted 6 August 2014

Academic Editor: Gongnan Xie

Copyright (C) 2015 Mohammad Ferdows et al. This is an open access article distributed under the Creative Commons Attribution License, which permits unrestricted use, distribution, and reproduction in any medium, provided the original work is properly cited.

Natural convective boundary-layer flow of a nanofluid on a heated vertical cylinder embedded in a nanofluid-saturated porous medium is studied. The model used for the nanofluid incorporates the effects of Brownian motion and thermophoresis. Lie groups analysis is used to get the similarity transformations, which transform the governing partial differential equations to a system of ordinary differential equations. Two groups of similarity transformations are obtained. Numerical solutions of the resulting ordinary differential systems are obtained and discussed for various values of the governing parameters.

\section{Introduction}

A nanofluid is a colloidal mixture of nanosized $(<100 \mathrm{~nm})$ particles in a base fluid. It is known that nanofluids can tremendously enhance the heat transfer characteristics of the original (base) fluid. Thus nanofluids have many applications in industry such as coolants, lubricants, heat exchangers, and microchannel heat sinks [1]. Nanoparticles have been made of various materials such as oxide ceramics and nitride ceramics. The objective of nanofluids is to achieve the best possible thermal properties with the least possible $(<1 \%)$ volume fraction of nanoparticles in the base fluid [1]. There have been many studies in the literature to better understand the mechanism behind the enhanced heat transfer characteristics.

There have been several recent experimental studies to better understand the mechanism of heat transfer enhancement during natural convection heat transfer in nanofluids $([2,3])$, and a summary can be found in Godson et al. [1]. Godson et al. [1] conclude that firm conclusions cannot be made from the limited number of studies and further experimental and theoretical work is required.
There have been several recent studies on the mathematical and numerical modelling of natural convection heat transfer in nanofluids by Congedo et al. [4]. Mathematical and numerical models have some advantages over experimental studies due to the many factors that influence nanofluid properties. Congedo et al. have used the computer code Fluent (release 6.3) to conduct numerical simulation of the water$\mathrm{Al}_{2} \mathrm{O}_{3}$ nanofluid. Natural convection in a horizontal tube heat exchanger was studied for a wide range of conditions and comparison made with available experimental data. Santra et al. [5] have used Artificial Neural Networks (ANN) to study heat transfer due to laminar natural convection of copperwater nanofluid in a differentially heated square cavity. The ANN was trained by resilient propagation algorithm. It was observed that the ANN was able to predict the heat transfer correctly within the given range of training data and is an efficient tool to predict heat transfer with reasonable computational time. Ho et al. [6] have used the finite volume approach with QUICK and central differencing for the convection and diffusion terms, respectively, to model natural convection of a nanofluid (alumina-water) in a square enclosure. They studied the effect of using two different formulas from 
the literature for effective viscosity and thermal conductivity of the nanofluid.

Lie group analysis, also called symmetry analysis, can be used to obtain similarity transformations that can be used to reduce the system of governing partial differential equations and associates boundary conditions to a system of ordinary differential equations. Ibrahim et al. [7] investigated the similarity reductions for problems of radiative and magnetic field effects on free convection and mass transfer flow past a semiinfinite flat plate. They obtained new similarity reductions and found an analytical solution for the uniform magnetic field case using Lie group method. They also presented numerical results for the nonuniform magnetic field case. Kalpakides and Balassas [8] studied the free convective boundary-layer problem of an electrically conducting fluid over an elastic surface by group theoretic method. Their results agree with the existing result for the group of scaling symmetry. They found that the numerical solution also does so. Megahed et al. [9] investigated convective heat and mass transfer along a semi-infinite vertical flat plate in the presence of a strong nonuniform magnetic field and the effects of Hall currents using the scaling group of transformations. They found that the temperature and concentration increase with an increase in magnetic parameter. Ibrahim et al. [10] presented symmetry group and similarity solutions for the steady laminar boundary-layer flow due to a rotating frustum of a cone in a viscous fluid. They found new four groups of similarity transformations. Hassanien and Hamad [11] introduced new similarity solutions of flow and heat transfer of a micropolar fluid along a vertical plate in a thermally stratified medium. The general analysis is developed in their study for the case of ambient temperature that varies exponentially with time, varies with the position, or has a uniform value. Some examples of the use of Lie group analysis to obtain similarity transformations for threedimensional Euler equations were presented by Sekhar and Sharma [12]. Bhuvaneswari et al. [13] presented similarity solutions of natural convection heat and mass transfer in an inclined surface with chemical reaction via Lie group analysis. Using symmetry analysis, Sahin et al. [14] studied the self-similarity solutions of the one-layer shallow-water equations representing gravity currents.

In this paper, the similarity solution of natural convective boundary-layer flow on a vertical cylinder embedded in a nanofluid-saturated porous medium is studied. Two groups of similarity transformations are presented.

\section{Analysis}

Consider steady two-dimensional free convection boundarylayer flow past a vertical circular cylinder of radius $L$ placed in a nanofluid-saturated porous medium. Cylindrical system of coordinates $(\bar{z}, \phi, \bar{r})$ are used in which the $\bar{z}$-axis is aligned vertically upwards. The temperature $T$ and the nanoparticle fraction $C$ take constant values $T_{w}$ and $C_{w}$ at the surface of the cylinder, $\bar{r}=L$. The ambient values, attained as $\bar{r}$ tends to infinity, are denoted by $T_{\infty}$ and $C_{\infty}$, respectively.

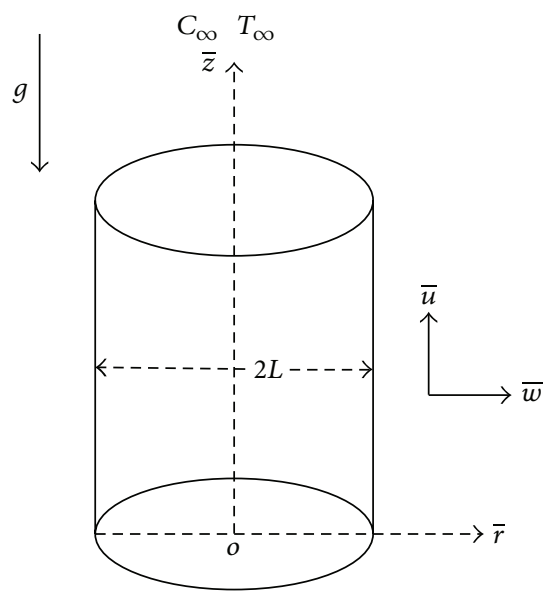

FIgURE 1: Physical model and coordinate system.

The Boussinesq approximation is employed. Homogeneity and local thermal equilibrium in the porous medium are assumed with porosity denoted by $\varepsilon$. The field variables are the velocity $\bar{V}$, the temperature $T$, and the nanoparticle volume fraction $C$. The following four field equations (in steady case) embody the conservation of total mass, momentum, thermal energy, and nanoparticles, respectively,

$$
\begin{gathered}
\nabla \cdot \bar{V}=0 \\
\nabla p+\frac{\mu}{k} \bar{V}=\left[\rho_{p} C+(1-C)\left\{\rho_{f}\left(1-\beta\left(T-T_{\infty}\right)\right)\right\}\right] g \\
(\rho c)_{f} \bar{V} \cdot \nabla T=k_{m} \nabla^{2} T \\
+\varepsilon(\rho c)_{p}\left[D_{B} \nabla C \cdot \nabla T+\left(\frac{D_{T}}{T_{\infty}}\right) \nabla T \cdot \nabla T\right] \\
\bar{V} \cdot \nabla C=\varepsilon D_{B} \nabla^{2} C+\varepsilon\left(\frac{D_{T}}{T_{\infty}}\right) \nabla^{2} T .
\end{gathered}
$$

Here $\rho_{f}$ is the density of the base fluid and $\mu, k$, and $\beta$ are the viscosity, thermal conductivity, and volumetric volume expansion coefficient of the nanofluid, respectively, while $\rho_{p}$ is the density of the particles. The porosity $\varepsilon$, the effective heat capacity $(\rho c)_{m}$, and the effective thermal conductivity $k_{m}$ of the porous medium are introduced. The gravitational acceleration is denoted by $g$. The coefficients that appear in (3) and (4) are the Brownian diffusion coefficient $D_{B}$ and the thermophoretic diffusion coefficient $D_{T}$. We consider a cylindrical polar coordinate system $(\bar{z}, \phi, \bar{r})$ corresponding to the axial, azimuthal, and radial directions, respectively, and denote the associated fluid velocities as $(\bar{u}, \bar{v}, \bar{w})$; the configuration is shown in Figure 1.

The boundary conditions are taken to be

$$
\begin{gathered}
\bar{w}=0, \quad T=T_{w}, \quad C=C_{w} \quad \text { at } \bar{r}=L, \\
\bar{u} \longrightarrow 0, \quad \bar{w} \longrightarrow 0, \quad T \longrightarrow T_{\infty}, \quad C \longrightarrow C_{\infty} \\
\text { as } \bar{r} \longrightarrow \infty,
\end{gathered}
$$


with the observation that the flow is axisymmetric so that $\bar{v}=0$ and all other dependent variables are independent of $\phi$, and, by making the standard boundary-layer approximation, based on a scale analysis, the governing equations (1)-(4) take the form

$$
\begin{gathered}
\frac{\partial}{\partial \bar{z}}(\bar{r} \bar{u})+\frac{\partial}{\partial \bar{r}}(\bar{r} \bar{w})=0 \\
\frac{\partial p}{\partial \bar{z}}=-\frac{\mu}{\bar{k}} \bar{u}+\rho_{f \infty} \beta g\left(1-C_{\infty}\right)\left(T-T_{\infty}\right) \\
-g\left(\rho_{p}-\rho_{f \infty}\right)\left(C-C_{\infty}\right) \\
\overline{u T}+\overline{\frac{\partial p}{\partial \bar{z}}}=0 \\
+\frac{\partial T}{\partial \bar{r}}=\alpha_{m} \frac{1}{\bar{r}} \frac{\partial}{\partial \bar{r}}\left(\bar{r} \frac{\partial T}{\partial \bar{r}}\right) \\
\left.+\bar{\tau} \frac{\partial C}{\partial \bar{z}}+\overline{D_{B}} \frac{\partial T}{\partial \bar{r}} \frac{\partial C}{\partial \bar{r}}+\frac{D_{T}}{T_{\infty}}\left(\frac{\partial T}{\partial \bar{r}}\right)^{2}\right] \\
+\varepsilon \frac{D_{T}}{T_{\infty}} \frac{1}{\bar{r}} \frac{\partial}{\partial \bar{r}}\left(\bar{r} \frac{\partial T}{\partial \bar{r}}\right),
\end{gathered}
$$

where

$$
\alpha_{m}=\frac{k_{m}}{(\rho c)_{f}}, \quad \tau=\frac{\varepsilon(\rho c)_{p}}{(\rho c)_{f}} .
$$

By eliminating $p$ from (7) and (8) by cross-differentiation, and by introducing the following nondimensional variables:

$$
\begin{gathered}
r=\frac{\bar{r}}{L}, \quad z=\frac{\bar{z}}{L \mathrm{Ra}_{L}}, \quad u=\frac{L}{\alpha \mathrm{Ra}_{L}} \bar{u}, \\
w=\frac{L}{\alpha} \bar{w}, \quad \theta=\frac{T-T_{\infty}}{T_{w}-T_{\infty}}, \\
\phi=\frac{C-C_{\infty}}{C_{w}-C_{\infty}}, \\
\operatorname{Ra}_{L}=\frac{k \rho_{f \infty} \beta g\left(1-C_{\infty}\right)\left(T_{w}-T_{\infty}\right) L}{\alpha \mu},
\end{gathered}
$$

where $\mathrm{Ra}_{L}$ is the Rayleigh number, (6)-(10) become in nondimensional form:

$$
\begin{gathered}
\frac{\partial}{\partial z}(r u)+\frac{\partial}{\partial r}(r w)=0 \\
\frac{\partial u}{\partial r}=\frac{\partial \theta}{\partial r}-\mathrm{Nr} \frac{\partial \phi}{\partial r} \\
u \frac{\partial \theta}{\partial z}+w \frac{\partial \theta}{\partial r}=\frac{1}{r} \frac{\partial}{\partial r}\left(r \frac{\partial \theta}{\partial r}\right)+\mathrm{Nb} \frac{\partial \theta}{\partial r} \frac{\partial \phi}{\partial r}+\mathrm{Nt}\left(\frac{\partial \theta}{\partial r}\right)^{2}, \\
\operatorname{Le}\left(u \frac{\partial \phi}{\partial z}+w \frac{\partial \phi}{\partial r}\right)=\frac{1}{r} \frac{\partial}{\partial r}\left(r \frac{\partial \phi}{\partial r}\right)+\frac{\mathrm{Nt}}{\mathrm{Nb}} \frac{1}{r} \frac{\partial}{\partial r}\left(r \frac{\partial \theta}{\partial r}\right),
\end{gathered}
$$

with the boundary conditions

$$
\begin{aligned}
& r=1: \quad w=0, \quad \theta=1, \quad \phi=1, \\
& r \longrightarrow \infty: \quad u \longrightarrow 0, \quad w \longrightarrow 0, \quad \theta \longrightarrow 0, \quad \phi \longrightarrow 0,
\end{aligned}
$$

where the parameters in (13) are defined by

$$
\begin{gathered}
\text { Le }=\frac{\alpha_{m}}{\varepsilon D_{B}}, \quad \mathrm{Nb}=\frac{\tau D_{B}\left(C_{w}-C_{\infty}\right)}{\alpha_{m}}, \\
\mathrm{Nt}=\frac{\tau D_{T}\left(C_{w}-C_{\infty}\right)}{\alpha_{m} T_{\infty}}, \\
\mathrm{Nr}=\frac{\left(\rho_{p}-\rho_{f \infty}\right)\left(C_{w}-C_{\infty}\right)}{\rho_{f \infty} \beta\left(1-C_{\infty}\right)\left(T_{w}-T_{\infty}\right)} .
\end{gathered}
$$

Here $\mathrm{Nr}, \mathrm{Nb}, \mathrm{Nt}$, and Le denote a buoyancy ratio, a Brownian motion parameter, a thermophoresis parameter, and a Lewis number, respectively.

We define the stream function $\psi$ according to

$$
u=\frac{1}{r} \frac{\partial \psi}{\partial r}, \quad w=-\frac{1}{r} \frac{\partial \psi}{\partial z}
$$

Using (16), (13)-(14) become

$$
\begin{gathered}
\frac{\partial}{\partial r}\left(\frac{1}{r} \frac{\partial \psi}{\partial r}\right)=\frac{\partial \theta}{\partial r}-\mathrm{Nr} \frac{\partial \phi}{\partial r}, \\
\frac{1}{r}\left(\frac{\partial \psi}{\partial r} \frac{\partial \theta}{\partial z}-\frac{\partial \psi}{\partial z} \frac{\partial \theta}{\partial r}\right) \\
=\frac{1}{r} \frac{\partial}{\partial r}\left(r \frac{\partial \theta}{\partial r}\right)+\mathrm{Nb} \frac{\partial \theta}{\partial r} \frac{\partial \phi}{\partial r}+\mathrm{Nt}\left(\frac{\partial \theta}{\partial r}\right)^{2}, \\
\operatorname{Le} \frac{1}{r}\left(\frac{\partial \psi}{\partial r} \frac{\partial \phi}{\partial z}-\frac{\partial \psi}{\partial z} \frac{\partial \phi}{\partial r}\right) \\
=\frac{1}{r} \frac{\partial}{\partial r}\left(r \frac{\partial \phi}{\partial r}\right)+\frac{\mathrm{Nt}}{\mathrm{Nb}} \frac{1}{r} \frac{\partial}{\partial r}\left(r \frac{\partial \theta}{\partial r}\right),
\end{gathered}
$$

with the boundary conditions

$$
\begin{aligned}
& r=1: \quad \frac{\partial \psi}{\partial z}=0, \quad \theta=1, \quad \phi=1 \\
& r \longrightarrow \infty: \quad \frac{\partial \psi}{\partial r} \longrightarrow 0, \quad \frac{\partial \psi}{\partial z} \longrightarrow 0, \quad \theta \longrightarrow 0, \quad \phi \longrightarrow 0
\end{aligned}
$$




\section{Symmetry Groups}

The symmetry groups of (17) are calculated using classical Lie group approach. The one-parameter infinitesimal Lie group of transformations leaving (17) invariant is defined as

$$
\begin{aligned}
& r * r+\varepsilon \xi_{1}(r, z, \psi, \theta, \phi), \\
& z * z+\varepsilon \xi_{2}(r, z, \psi, \theta, \phi), \\
& \psi * \psi+\varepsilon \mu_{1}(r, z, \psi, \theta, \phi), \\
& \theta * \theta+\varepsilon \mu_{2}(r, z, \psi, \theta, \phi), \\
& \phi * \phi+\varepsilon \mu_{3}(r, z, \psi, \theta, \phi),
\end{aligned}
$$

and the infinitesimal generator has the following form:

$$
\begin{aligned}
X= & \xi_{1}(r, z, \psi, \theta, \phi) \frac{\partial}{\partial r}+\xi_{2}(r, z, \psi, \theta, \phi) \frac{\partial}{\partial z} \\
& +\mu_{1}(r, z, \psi, \theta, \phi) \frac{\partial}{\partial \psi}+\mu_{2}(r, z, \psi, \theta, \phi) \frac{\partial}{\partial \theta} \\
& +\mu_{3}(r, z, \psi, \theta, \phi) \frac{\partial}{\partial \phi} .
\end{aligned}
$$

By carrying out a straightforward and tedious algebra, we finally obtain the form of the infinitesimals $\xi_{1}, \xi_{2}, \mu_{1}, \mu_{2}$, and $\mu_{3}$ as

$$
\begin{gathered}
\xi_{1}=\frac{1}{2} a_{1} r, \quad \xi_{2}=a_{1} z+a_{2}, \quad \mu_{1}=a_{1} \psi+a_{3}, \\
\mu_{2}=a_{4}, \quad \mu_{3}=a_{5},
\end{gathered}
$$

where $a_{1}, a_{2}, a_{3}, a_{4}$, and $a_{5}$ are constants. The infinitesimals generators that correspond to the infinitesimals (21) are

$$
\begin{aligned}
& X_{1}=\frac{1}{2} r \frac{\partial}{\partial r}+z \frac{\partial}{\partial z}+\psi \frac{\partial}{\partial \psi} \\
& X_{2}=\frac{\partial}{\partial z}, \quad X_{3}=\frac{\partial}{\partial \psi}, \\
& X_{4}=\frac{\partial}{\partial \theta}, \quad X_{5}=\frac{\partial}{\partial \phi} .
\end{aligned}
$$

Further details on similarity transformations using Lie group analysis can be found in [15-18].

\section{Reductions to Ordinary Differential Equations}

We will produce the similarity transformations and solutions using infinitesimal generators given in (22). Imposing the restrictions from the boundary conditions, we choose only the generator $X_{1}$.

The characteristic functions are

$$
\frac{d r}{r / 2}=\frac{d z}{z}=\frac{d \psi}{\psi}=\frac{d \theta}{0}=\frac{d \phi}{0},
$$

from which the independent similarity variable, the stream function, the temperature, and the mass fraction turn out to be of the form

$$
\eta=\frac{r}{\sqrt{z}}, \quad \psi=z F(\eta), \quad \theta=\theta(\eta), \quad \phi=\phi(\eta) .
$$

Substituting (24) into the partial differential equations (17)(18), we finally obtain the system of nonlinear ordinary differential equations:

$$
\begin{gathered}
\eta F^{\prime \prime}-F^{\prime}-\eta^{2}\left(\theta^{\prime}-\mathrm{Nr} \phi^{\prime}\right)=0, \\
\eta \theta^{\prime \prime}+(1+F) \theta^{\prime}+\mathrm{Nb} \eta \theta^{\prime} \phi^{\prime}+\mathrm{Nt} \eta \theta^{\prime 2}=0, \\
\eta \phi^{\prime \prime}+(1+\operatorname{Le} F) \phi^{\prime}+\frac{\mathrm{Nt}}{\mathrm{Nb}}\left(\eta \theta^{\prime \prime}+\theta^{\prime}\right)=0 .
\end{gathered}
$$

The appropriate boundary conditions are expressed as

$$
\begin{aligned}
& \eta=\zeta: \quad F=\frac{1}{2} \zeta F^{\prime}, \quad \theta=1, \quad \phi=1, \\
& \eta \longrightarrow \infty: \quad F^{\prime} \longrightarrow 0, \quad \theta \longrightarrow 0, \quad \phi \longrightarrow 0,
\end{aligned}
$$

where primes denote differentiation with respect to $\eta$, and $\zeta=$ $z^{-1 / 2}$.

Now, if we consider $\eta=(r-1) / \sqrt{z}$ instead of $\eta=r / \sqrt{z}$ which are given in (24), the similarity representation will be as follows:

$$
\begin{gathered}
(\eta+\zeta) F^{\prime \prime}-F^{\prime}-\left(\eta^{2}+2 \zeta \eta+\zeta^{2}\right)\left(\theta^{\prime}-\mathrm{Nr} \phi^{\prime}\right)=0 \\
(\eta+\zeta) \theta^{\prime \prime}+(1+F) \theta^{\prime} \\
+\mathrm{Nb}(\eta+\zeta) \theta^{\prime} \phi^{\prime}+\mathrm{Nt}(\eta+\zeta) \theta^{\prime 2}=0, \\
(\eta+\zeta) \phi^{\prime \prime}+(1+\operatorname{Le} F) \phi^{\prime}+\frac{\mathrm{Nt}}{\mathrm{Nb}}\left[(\eta+\zeta) \theta^{\prime \prime}+\theta^{\prime}\right]=0
\end{gathered}
$$

with the boundary conditions

$$
\begin{aligned}
& \eta=0: \quad F=0, \quad \theta=1, \quad \phi=1, \\
& \eta \longrightarrow \infty: \quad F^{\prime} \longrightarrow 0, \quad \theta \longrightarrow 0, \quad \phi \longrightarrow 0 .
\end{aligned}
$$

\section{Results and Discussions}

The similarity solution of free convective boundary-layer flow on a vertical cylinder embedded in a nanofluid-saturated porous medium is obtained. Two groups of similarity transformations and the corresponding ordinary differential systems are presented.

The two systems (25)-(26) and (27)-(28) have been solved numerically by using finite difference method (MATLAB package). The numerical solutions of the first system (25)(26) are shown in Figures 2 and 3, while Figures 4 and 5 represent the numerical solutions of the second system (27)(28). In first system we considered $\zeta=1$, while in the second system we put $\zeta=0$ ( $\zeta$ arbitrary). 


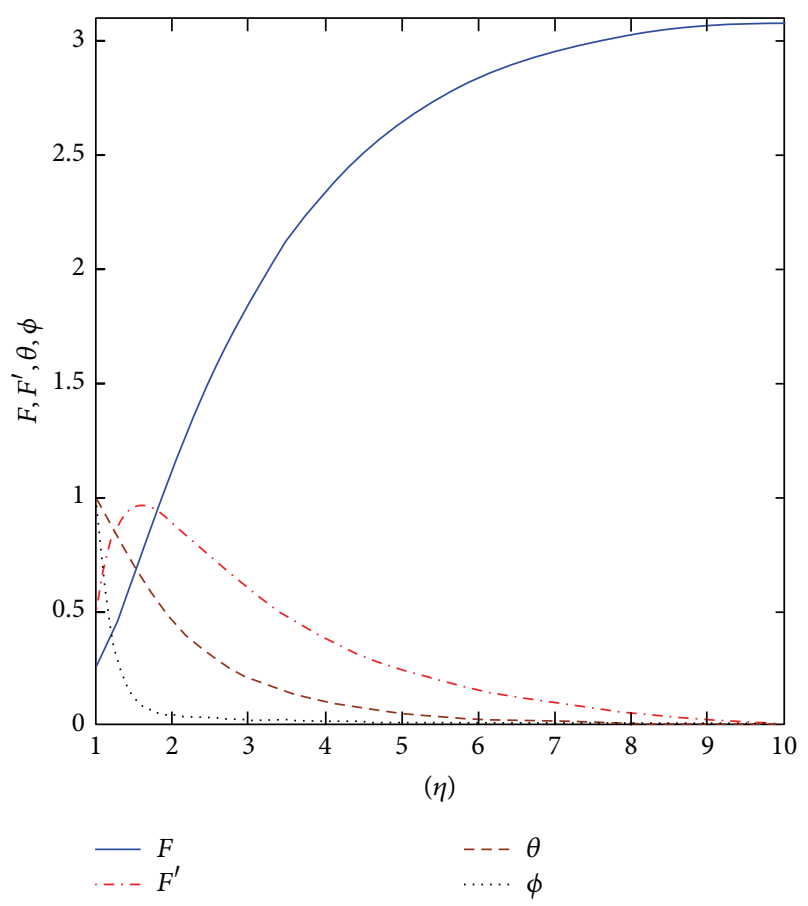

FIgURE 2: Plots of dimensionless similarity functions $F(\eta), F^{\prime}(\eta)$, $\theta(\eta)$, and $\phi(\eta)$ for the case of $\mathrm{Le}=10, \mathrm{Nr}=0.5, \mathrm{Nb}=0.5, \mathrm{Nt}=0.5$, and $\zeta=1$.

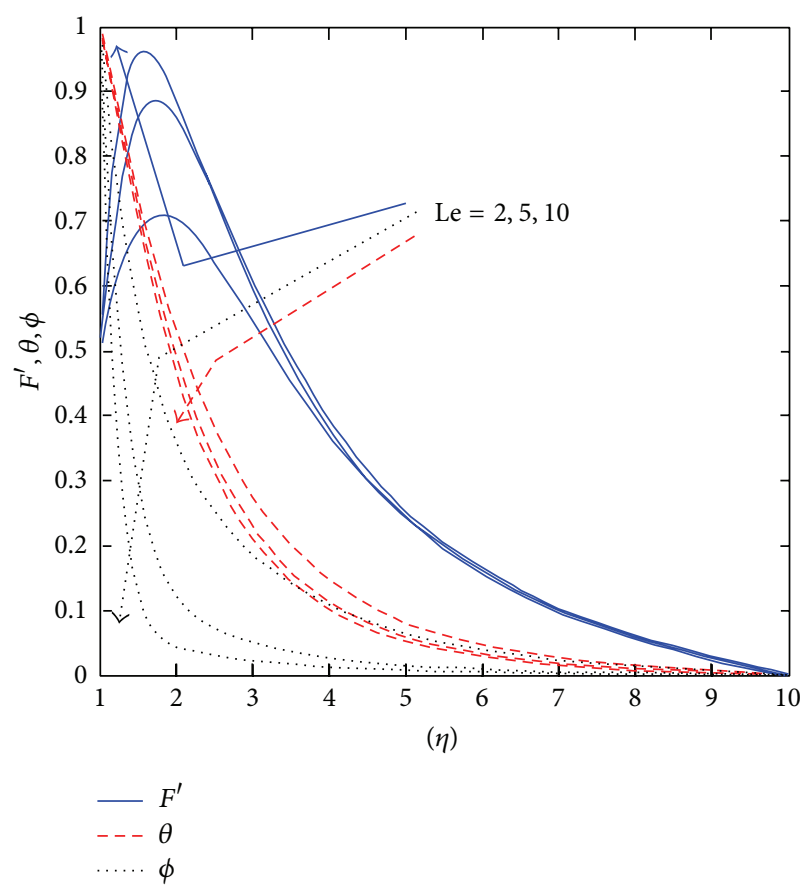

FIgURE 3: Plots of dimensionless similarity functions $F^{\prime}(\eta), \theta(\eta)$, and $\phi(\eta)$ for various values of Le at $\mathrm{Nr}=0.5, \mathrm{Nb}=0.5, \mathrm{Nt}=0.5$, and $\zeta=1$.

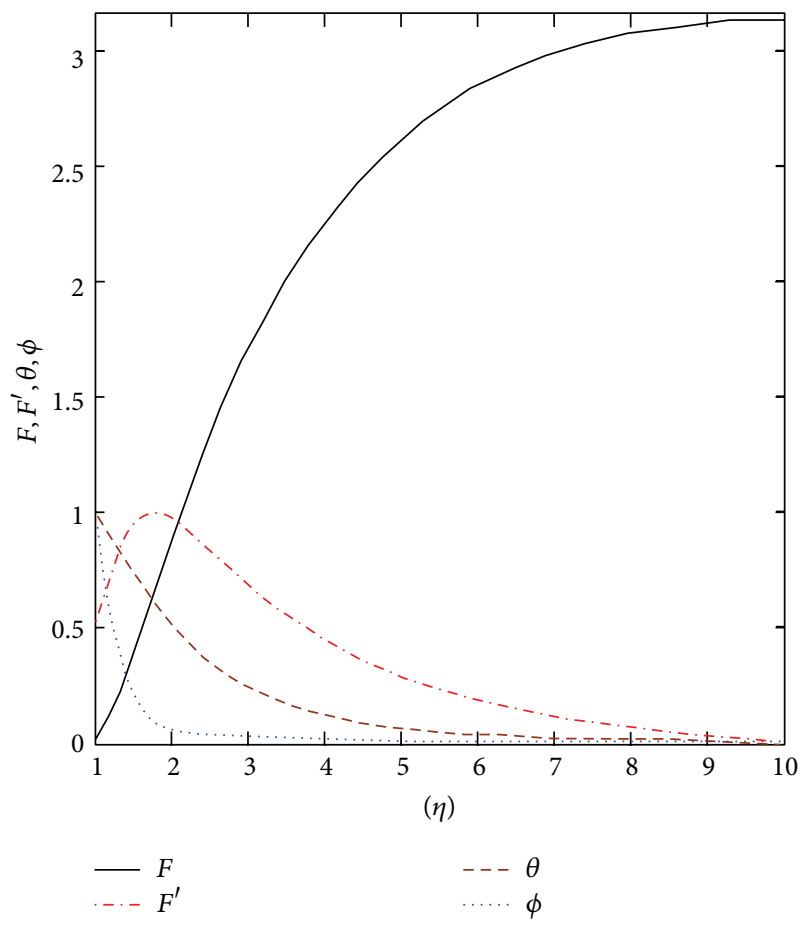

Figure 4: Plots of dimensionless similarity functions $F(\eta), F^{\prime}(\eta)$, $\theta(\eta)$, and $\phi(\eta)$ for the case of $\mathrm{Le}=10, \mathrm{Nr}=0.5, \mathrm{Nb}=0.5, \mathrm{Nt}=0.5$, and $\zeta=0$.

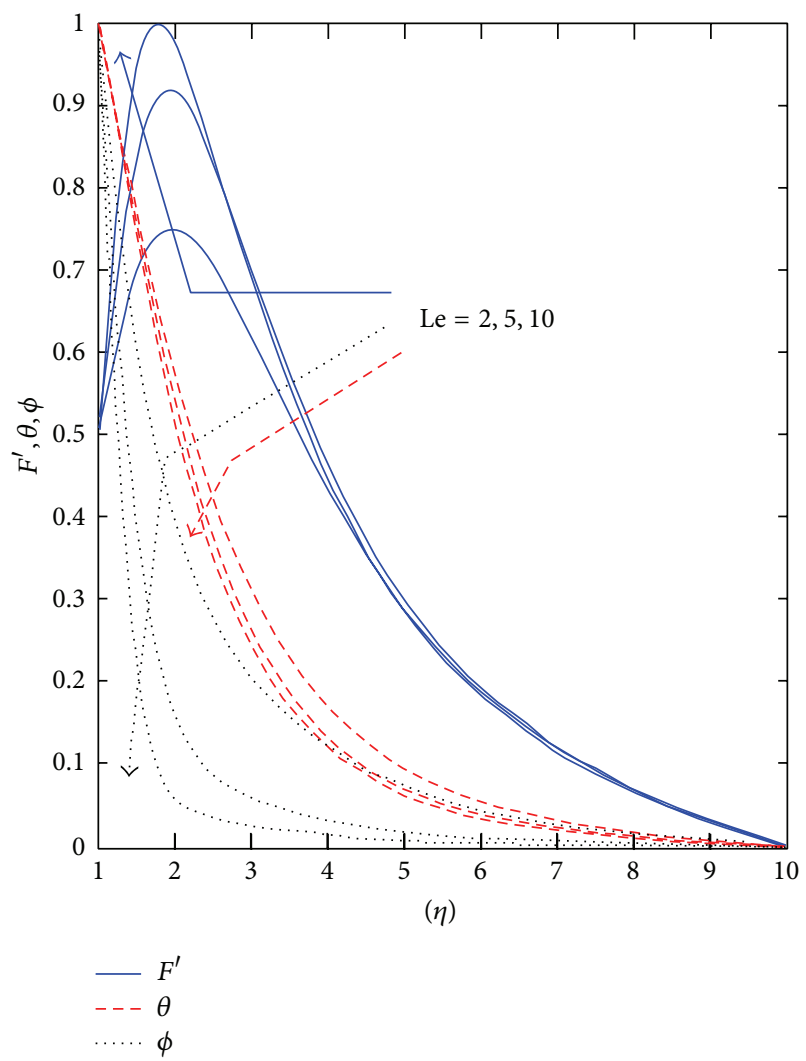

Figure 5: Plots of dimensionless similarity functions $F^{\prime}(\eta), \theta(\eta)$, and $\phi(\eta)$ for various values of Le at $\mathrm{Nr}=0.5, \mathrm{Nb}=0.5, \mathrm{Nt}=0.5$, and $\zeta=0$. 
Plots of the dependent similarity variables for $\mathrm{Le}=10, \mathrm{Nr}$ $=0.5, \mathrm{Nb}=0.5$, and $\mathrm{Nt}=0.5$ for both systems are shown in Figures 2 and 4 [19]. We have found that the boundary-layer profiles for the stream function $F(\eta)$ and the temperature function $\theta(\eta)$ have essentially the same form as in the case of a regular fluid. The thickness of the boundary-layer for the mass fraction function $\phi(\eta)$ is smaller than the thermal boundary-layer thickness when Le $>1$. We notice that in the case of a nanofluid the two profiles of the velocity $F^{\prime}(\eta)$ and the temperature $\theta$ diverge within a layer whose thickness is comparable with that of the mass fraction.

Figure 3 shows the behavior of the velocity $F^{\prime}(\eta)$, the temperature $\theta(\eta)$, and the mass fraction function $\phi(\eta)$ for various values of Lewis number Le when $\mathrm{Nr}=0.5, \mathrm{Nb}=0.5$, $\mathrm{Nt}=0.5$, and $\zeta=1$ (first system). It is observed that the velocity $F^{\prime}(\eta)$ increases as the Lewis number Le increases. Also, it is noticed that the thicknesses of the thermal and the mass fraction boundary layers are decreased with increasing Le.

Figure 5 displays the effect of Lewis number Le on the velocity, the temperature, and the nanoparticle volume fraction for $\mathrm{Nr}=0.5, \mathrm{Nb}=0.5, \mathrm{Nt}=0.5$, and $\zeta=0$ (second system). For the second system we have gotten the same behavior as in Figure 4 (numerical from system 1).

\section{Conclusions}

The symmetries of the governing system of partial differential equations are obtained using Lie group analysis and they reduce the system to system of ordinary differential equations. The similarity solution depends on four dimensionless parameters, namely, a Lewis number Le, a buoyancy-ratio parameter $\mathrm{Nr}$, a Brownian motion parameter $\mathrm{Nb}$, and a thermophoresis parameter Nt. From the numerical results, the thickness of the thermal boundary layer is greater than the thickness of the mass fraction boundary layer when Le $>1$. In the case of a nanofluid the two profiles of the velocity $F^{\prime}(\eta)$ and the temperature $\theta$ diverge within a layer whose thickness is comparable with that of the mass fraction.

\section{Conflict of Interests}

The authors declare that there is no conflict of interests regarding the publication of this paper.

\section{Acknowledgment}

The authors extend their appreciation to the Deanship of Scientific Research at King Saud University for funding this work through the research group Project no. RGP-VPP-080.

\section{References}

[1] L. Godson, B. Raja, D. Mohan Lal, and S. Wongwises, "Enhancement of heat transfer using nanofluids-an overview," Renewable and Sustainable Energy Reviews, vol. 14, no. 2, pp. 629-641, 2010.

[2] N. Putra, W. Roetzel, and S. K. Das, "Natural convection of nano-fluids," International Journal of Heat and Mass Transfer, vol. 39, no. 8-9, pp. 775-784, 2003.
[3] D. Wen and Y. Ding, "Formulation of nanofluids for natural convective heat transfer applications," International Journal of Heat and Fluid Flow, vol. 26, no. 6, pp. 855-864, 2005.

[4] P. M. Congedo, S. Collura, and P. M. Congedo, "Modeling and analysis of natural convection heat transfer in nanofluids," in Proceedings of the ASME Summer Heat Transfer Conference, vol. 3, pp. 569-579, August 2008.

[5] A. K. Santra, N. Chakraborty, and S. Sen, "Prediction of heat transfer due to presence of copper-water nanofluid using resilient-propagation neural network," International Journal of Thermal Sciences, vol. 48, no. 7, pp. 1311-1318, 2009.

[6] C. J. Ho, M. W. Chen, and Z. W. Li, "Numerical simulation of natural convection of nanofluid in a square enclosure: effects due to uncertainties of viscosity and thermal conductivity," International Journal of Heat and Mass Transfer, vol. 51, no. 1718, pp. 4506-4516, 2008.

[7] F. S. Ibrahim, M. A. Mansour, and M. A. A. Hamad, "Liegroup analysis of radiative and magnetic field effects on free convection and mass transfer flow past a semi-infinite vertical flat plate," Electronic Journal of Differential Equations, vol. 39, pp. $1-17,2005$.

[8] V. K. Kalpakides and K. G. Balassas, "Symmetry groups and similarity solutions for a free convective boundary-layer problem," International Journal of Non-Linear Mechanics, vol. 39, no. 10, pp. 1659-1670, 2004.

[9] A. A. Megahed, S. R. Komy, and A. A. Afify, "Similarity analysis in magnetohydrodynamics: hall effects on free convection flow and mass transfer past a semi-infinite vertical flat plate," International Journal of Non-Linear Mechanics, vol. 38, no. 4, pp. 513-520, 2003.

[10] F. S. Ibrahim, M. A. Mansour, and M. A. A. Hamad, "Similarity solution of laminar flow due to a rotating frustum of a cone," Journal of the Egyptian Mathematical Society, vol. 15, pp. 1-13, 2007.

[11] I. A. Hassanien and M. A. A. Hamad, "Group theoretic method for unsteady free convection flow of a micropolar fluid along a vertical plate in a thermally stratified medium," Applied Mathematical Modelling, vol. 32, no. 6, pp. 1099-1114, 2008.

[12] T. R. Sekhar and V. D. Sharma, "Similarity solutions for three dimensional Euler equations," Applied Mathematics and Computation, vol. 196, no. 1, pp. 147-157, 2008.

[13] M. Bhuvaneswari, S. Sivasankaran, and M. Ferdows, "Lie group analysis of natural convection heat and mass transfer in an inclined surface with chemical reaction," Nonlinear Analysis. Hybrid Systems, vol. 3, no. 4, pp. 536-542, 2009.

[14] D. Sahin, N. Antar, and T. Ozer, "Lie group analysis of gravity currents," Nonlinear Analysis: Real World Applications, vol. 11, no. 2, pp. 978-994, 2010.

[15] L. V. Ovsiannikov, Group Analysis of Differential Equations, Nauco, Moscow, Russia, 1978, English translation, W. F. Ames, Academic Press, New York, NY, USA, 1982.

[16] P. J. Olver, Applications of Lie Groups to Differential Equations, vol. 107 of Graduate Texts in Mathematics, Springer, Berlin, Germany, 2nd edition, 1993.

[17] N. H. Ibragimov, CRC Handbook of Lie Group Analysis to Differential Equations, CRC Press, 1994.

[18] G. W. Bluman and S. Kumei, Symmetries and Differential Equations, Springer, New York, NY, USA, 1989.

[19] A. Bagheri, H. Gholami, and M. Maghsoudi, "Natural convective boundary layer flow of a nanofluid past a vertical plate with heat flux condition," International Research Journal of Applied and Basic Sciences, vol. 5, pp. 1406-1414, 2013. 


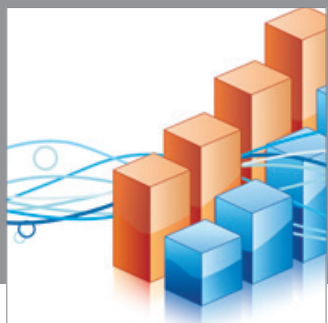

Advances in

Operations Research

mansans

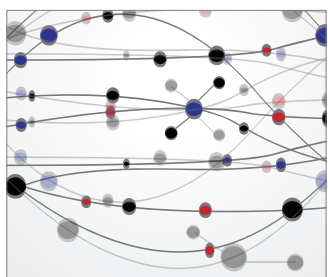

The Scientific World Journal
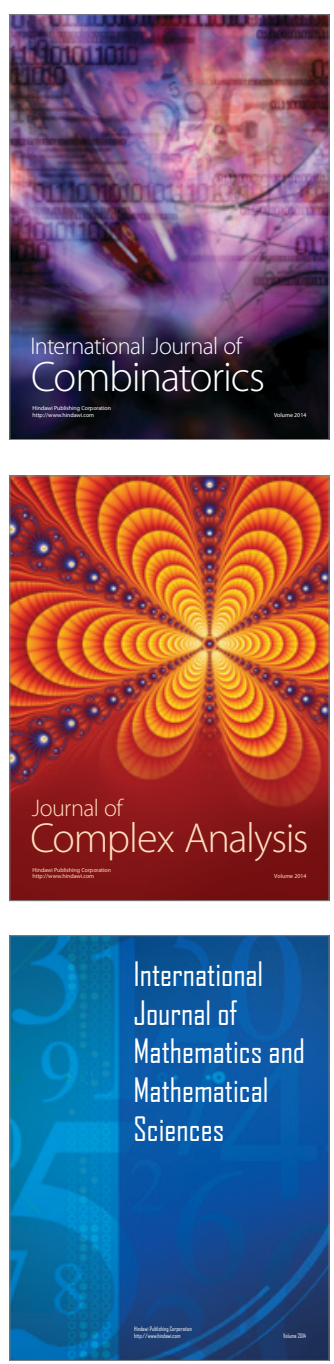
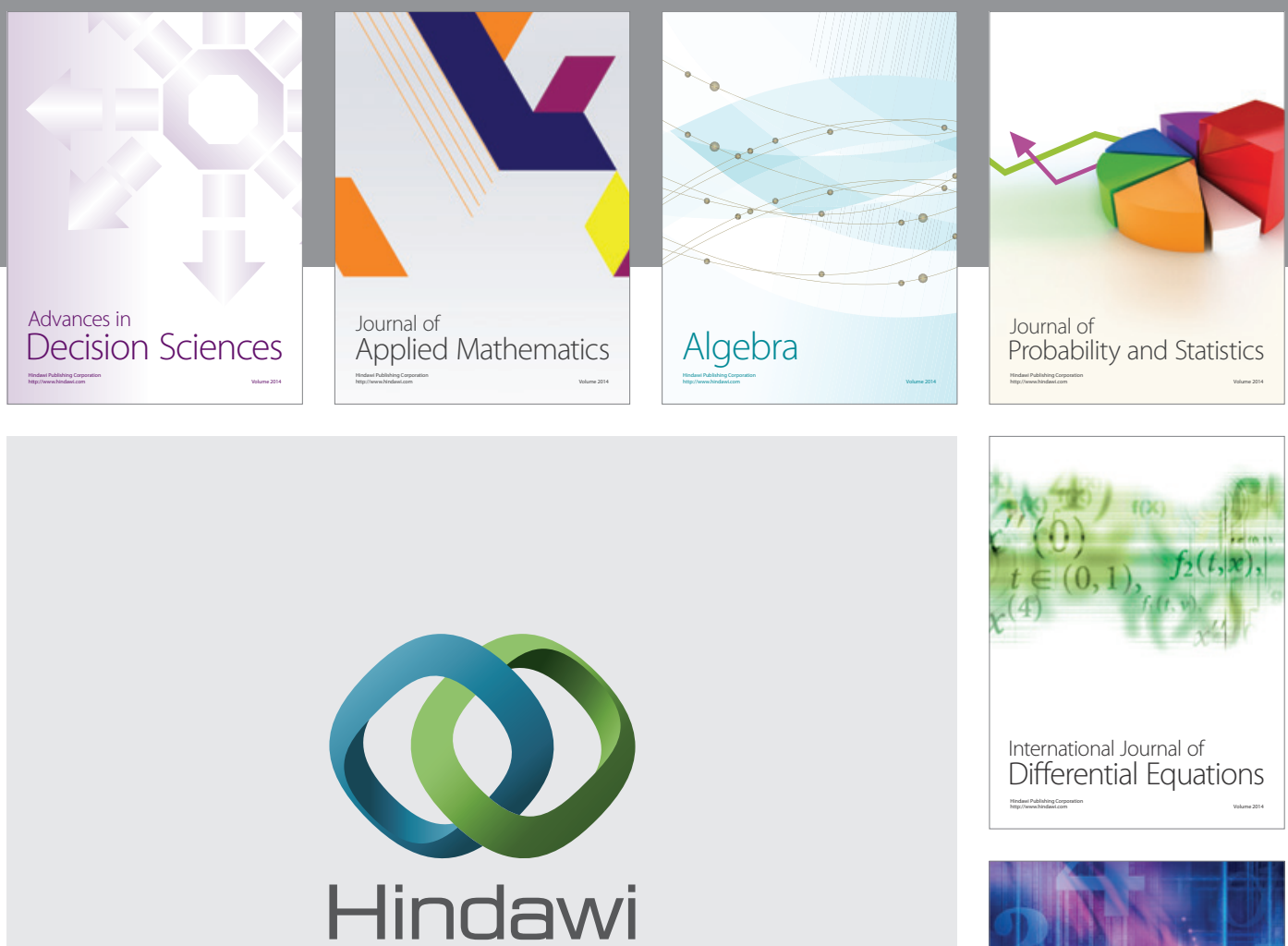

Submit your manuscripts at http://www.hindawi.com
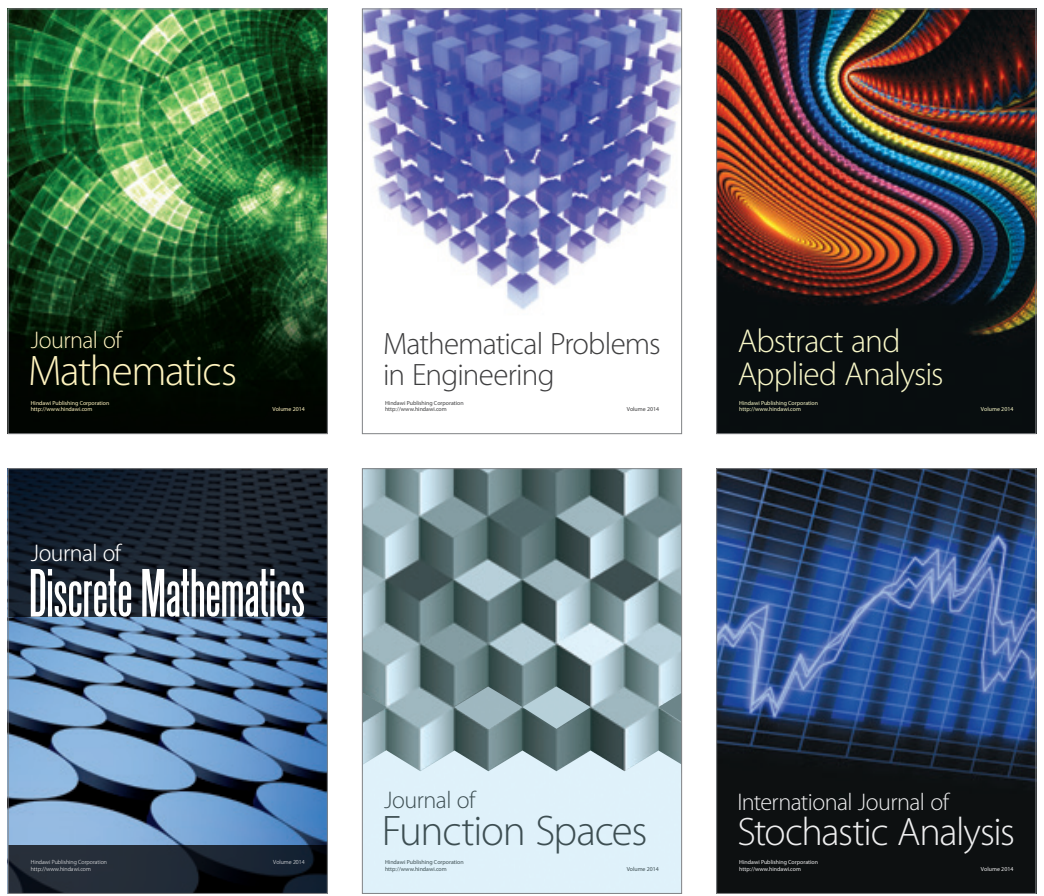

Journal of

Function Spaces

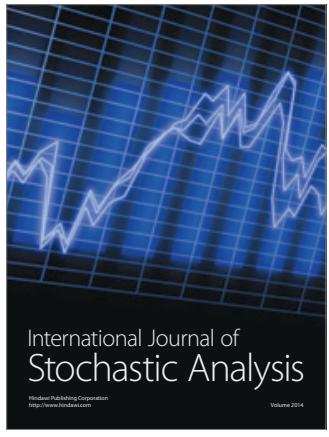

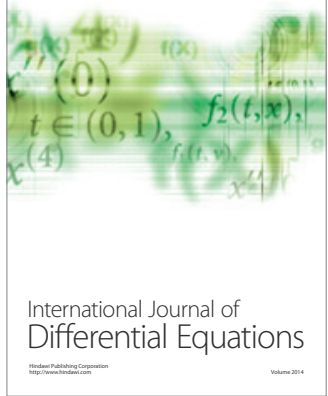
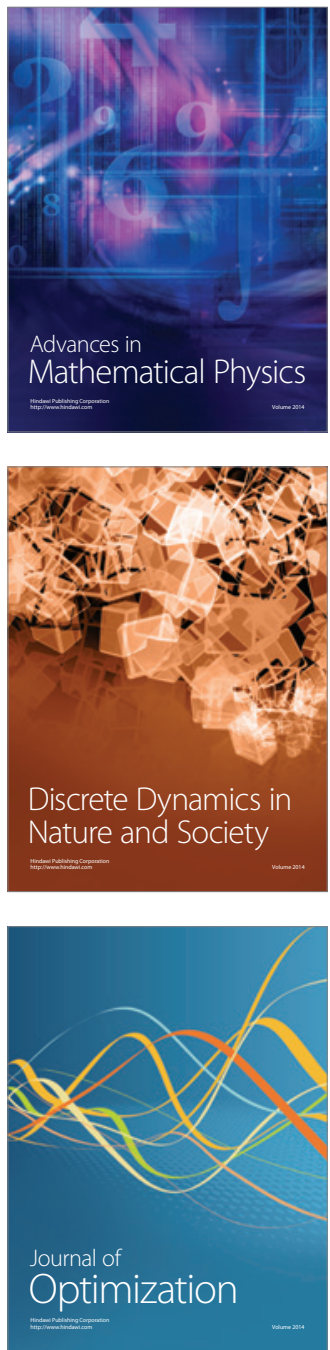